\title{
Prospects in electronic publishing
}

\author{
Reports from the meeting on electronic \\ publishing held by the relevant \\ committee of the International \\ Publishers Association in Frankfurt, \\ Germany, in September 1992.
}

\section{Introduction: Gérard Lamy}

In the last four years we have brought to this assembly a knowledge of what were the new tools for the electronic industry: SGML, CD-ROM, Audio Books, electronic books or hand-held computers, using the 'solid state' or the CD-ROM technology, CDI, desktop publishing, network publishing. One of the intended goals of our Committee is to make publishers aware of the new technologies, which will in a near future bring new tools to the publishing industry.

The last three years have brought to our industry some very important new tools. Gone is the time when publishers were asking manufacturers to produce affordable and easy to use hardware and manufacturers were asking publishers to conceive good editorial products adapted to the Electronic publishing market.

Many times here we have reminded this assembly, how Electronic Publishing could bring new ways of publishing and how on the other side, it could lead other industries to step into our market, if we were not fast enough. Look what happens to the Handheld CD-ROM when you see a manufacturer who is now a publisher!

As today is my last meeting as Chairman of our Committee, I cannot resist a look at the past, which I seldom do, and raise some questions. SGML: in New Delhi in January this year I said in my introduction that SGML [Standard Generalized/Generic Markup Language: Ed.] was widely known if not used as it should be. I am amazed to see so many publishers and not the smallest ones by far, who are still postponing the adoption of this standard. Others, who have decided to 'go on SGML' like going to prison, still weigh whether their editorial staff will be put in charge, or an outside firm, like a printer, will do the job! Among those who have adopted SGML, a significant number, after two or three years have failed. This only to illustrate how many publishers are considering new technologies, attached as they are to the centuries old paper, and how Management is so often afraid of investing time and money on such new ventures. I know publishers who make $50 \%$ of their profits from Electronic publishing.

In this assembly today, we have people who have been very successful by investing entirely in new technologies, and we have too, many who would like to see their Management doing what it should. In New Delhi this year, $\mathrm{Mr}$ Nicholas Negroponte, the Director of the MIT Media Laboratory, addressed our assembly with one of the best lectures I have heard on the future of Electronic Publishing. Mr Negroponte said that in the future (ten or fifteen years) everything will be digitalized, from texts, to sound, to pictures.

Any publisher with a mere $1000 \$$ scanner, can digitalize texts and pictures, and all use computers for editing texts. Mr Negroponte's message was: In the future all the production on paper today will have to be also delivered electronically.

In ten or fifteen years, our customers will be those in their teens today . . . the computer generation. Think of those CD-ROM Hand-helds storing 2000 million characters! Think also of the future screens, which we will have in three or four years - nothing in common with those we know today.

\section{Networks and publishers: Mari Pijnenborg}

We should, in fact, start with the end-user who is going to get access straight away to the network. Some conversion/transformation of the information has to take place. It is misleading to speak of a little conversion. It is 
important to realise that the digital library will have as a consequence that the location and formatting of the information will be irrelevant. The end-user will be equipped with a desktop personal library in which he will collect all information relevant to him whether for personal or functional use. Everything related to his projects, logistics, is simply packaged in a personal library to which he has desktop access.

It is important we deliver speed. We have to contribute to the productivity of the researcher. In terms of productivity it is no longer enough to avail information ${ }^{\star}$. Communication services must really contribute to the productivity of the creative and intellectual effort of the scientist, of the researcher.

From this moment we agree that our role is knowledge services rather than information services we have to accept the consequences that we will have to use information technology to enable the end-user to interact with the information providing him with the ability to move from information into knowledge services. Information Technology I would prefer to term Interactive Technology - between provider and user.

In Interactive Technology, if we are not very conscious of the fact that access to the end-user is very vital to our autonomy of entrepreneurship then in my judgement we are not doing the right thing.

Information communication will no longer be supply driven as in the past so we must look for our own authorship, in addition to that of authors, in creating these knowledge services. This means we must look for legal protection to make sure that the intellectual and financial efforts that we put into these knowledge-bases, into those 'decision support tools' are properly protected and in that respect copyright still remains an important instrument which, unfortunately, does not serve all requirements, e.g. the authorship of publishers must be protected as an independent right.

Only in adding value in the eyes of the end-users, only by intellectual packaging, only by development of knowledge-bases, can we remain competitive among ourselves and other parties in communication business.

\section{CD networks: Tony Feldman}

One of the features of CD-ROM which should have once made it an attractive medium for conventional publishers, was its 'uninterconnectedness'. In other words, CD-ROM as electronic book - discrete, stand-alone, ideal for use by one reader at a time. Today, the writing is on the wall for this cosy notion. The days of $C D$ information as a neat one-to-one affair are nearly over. CD information is going the way of all information and at last finding its way onto the networks.

What is the evidence for this? TFPL's CD-ROM Directory provides a clear indicator. In the 1991 edition, 1623 commercial CD-ROM titles were listed of which 213 (13.1 per cent) were quoted as having network prices fixed by their publishers. The 1992 edition lists 2212 titles including 419 multimedia discs. Out of the 1793 nonmultimedia CD-ROMs, there are 387 (22 per cent) with quoted network prices. In other words, the proportion of publishers at least addressing the networking issue has almost doubled in the last year.

It looks certain that the vast majority of networks are located in the United States and are being used largely in professional, academic and corporate libraries.

We need to ask how $C D$ publishers are responding. The TFPL statistics indicate that some are at least recognising the networking as an issue. However, if 22 per cent are currently quoting network prices, what are the other 78 per cent doing? In practice, unless publishers have taken deliberate steps to prevent it, discs can be put into a network and accessed simultaneously by a number of users either locally or, more worrying, across wide area networks via dial-in access.

Unless publishers start regulating the use of their CD-ROMs on networks, there is a real danger that the exchange and sharing of CD-ROM information could get out of control. One result of this would be a rapid drop in disc sales and, in response, a winding down of the publishing investment. Ultimately, network anarchy could undermine the commercial basis of the CD-ROM industry and so cut the throats of publishers and users alike.

In reality, of course, networking is not growing so quickly that such a nightmare scenario is imminent. But the danger exists, particularly because right now there seems to be only two kinds of CD-ROM publishers those in danger of missing the networking 
boat and those who do not even know there is a boat to catch.

On the other hand, a brief examination of the licensing policies suggests a patchwork of strategies offering customers little logic or coherence. Pricing policies alone are already causing irritation. Some library users, in particular, fume at the sheer inconsistencies they encounter. They point out that while some publishers impose high network license charges, others charge nothing. Resentment is growing and, if nothing is done, could eventually incite some users to ignore publishers interests altogether and to begin using $C D$ ROMs on networks, licensed or not.

The key question - one which may well determine the future of the CD-ROM publishing industry - is really very simple. Are publishers going to pluck their heads from the sand in time, see the signals and take action?

\section{Customized publishing on demand: Samuel J. Constanzo}

A powerful application of information technology that allows a teacher or end-users of any sort to fully customize their readings or course materials at the simplest level, Primis allows the reader to select material from a larger retrievable database which integrates different subject ideas and types of information which the users can then arrange in the manner that best suits their needs; response by McGraw-Hill to market demands for greater and more variable access to information resources and to the demand to segment or customize content to meet specific needs for academic markets around the world. Professors continually ask for a differnt organization of text materials and want to add material of their own. The proliferation of 'course packs' underscores this.

Primis is also a response to the new economics of publishing text materials.

It affords the opportunity to increase customer interaction by delivering material more quickly and in a more targeted, specific manner. Customer interaction will lead to increased author/editor response to readers and as a result, an increase in the quality and tailored diversity of the content.

- It creates an environment in which we, as publishers, can revise our content due solely to change in the content or in pedagogy.
Nothing is out of print and everything can remain available to our customers. It is a just-in-time manufacturing system that eliminates inventory and the costs associated with the management and administration of the inventory, eliminating out-of-stock and out-of-print books.

- It offers publishers the chance to sell their highly developed information on a subscription basis and on an ongoing consultative basis.

Finally, Primis is founded on the fundamental principle of multiplicity of use: the reorganization of an information base and the sale of that information into multiple markets. Only with Primis do we have the capability to reach all markets for our information resources, since we can tailor the delivery of that information to whatever format or setting is required.

Because the Primis system treats each item of the database (chapter, article, problem, paragraph) as if it were a book with its own identifying number (at McGraw-Hill that is an ISBN), it is fairly simple to track sales and hence, administer royalties or anything that is traditionally associated with books. Authors sign an agreement allowing McGraw-Hill to input their content into Primis, recombine their material in the manner allowed via Primis and to be printed, bound and sold. We have not created, nor have we received, the rights for (new) electronic data transmission over the Primis network. In short, the integrity of the current copyright law is maintained.

The Primis system has been set up to accommodate multiple publishers. Each publisher's own database is partitioned and separated from other published material. Within McGraw-Hill's database we have received permissions to include materials from nearly 100 publishers worldwide, both big and small, scholarly and trade. I believe we will move slowly in this industry to full cooperation and inclusion of licensed material.

McGraw-Hill intended from very early on in the development of Primis to make whatever systems or technology that are part of the Primis system available to all publishers. We believe we have such a system. When you license the entire Primis system (hardware system, software, order-entry, and dynamic index) McGraw-Hill services and trains users on the Order-Entry System and the Dynamic 
Index. It is also ready to provide important assistance in launching a database in this environment.

As an information services company we will compete and survive based upon the breadth and quality of our information resource - our content, our books and our database. Licensing Primis acknowledges this fact, and it also acknowledges that the information needs of our customers require, ultimately, the collaboration and cooperative efforts of all publishers. If we as an industry make every effort to satisfy our readers, we will control our destiny, increase sales and revenue, and better position ourselves for a changing environment.

\section{Standardizing the interchange of electronic documents: David Buckle}

During the past two years the library communities of North America and Europe have focused a great deal of their public debate upon the topic of networking. The prospect before the academic communities in Europe and North America is to move from a wide area network environment of $2 \mathrm{Mbps}$ capacity (JANET MKII) to an environment offering $622 \mathrm{Mbps}$ and rapid expansion to $2488 \mathrm{Mbps}$ (SUPER JANET) before the third quarter of the decade. In parallel local area networks will generally have capacity of $155 \mathrm{Mbps}$. Such monumental growth does not simply offer opportunity to do contemporary things better, it creates a novel environment of opportunity for all professional and social policy makers that have access to it and guardianship of it. This network environment will span continents, particularly Europe, the Pacific rim and North America.

To maximise the opportunity that this novel environment presents will demand change, particularly to the library and information professional's perspective of his environment, and the library and information suppliers perspective of his insularity in the market place. What were once clearly defined professional demarcation lines will become blurred and what once was pride of commercial independence will become constructive interdependence.

The Group on Electronic Document Interchange (GEDI) was established in October 1990. The resulting statement of its proposals defines a mutually accepted technical framework to facilitate electronic document interchange between the GEDI partners. Those partners include: The British Library Document Supply Centre (UK), Ministère de l'Education Nationale, Sous-Direction des Bibliothèques, Questel, Télésystèmes (France), PICA Centrum voor Bibliotheekautomaatisering (Netherlands), Technische Informationsbibliothek (TIB) Hannover (Germany), OCLC Online Computer Library Center Inc. (USA), Research Libraries Group (RLG: USA). All have a mutual interest in the electronic exchange of the full texts of documents. For those with a thirst for more detailed knowledge, I commend them to the GEDI report: Electronic Document Delivery Towards Further Standardisation of International Interchange. Proposals of the Group on Electronic Document Interchange (GEDI) Version 10 September, 1991. Available from PICA, Leiden, Netherlands.

Within twelve months of an idea the GEDI group was able to formulate a framework of standards which facilitate interconnection between discrete systems. Within a year of publishing their proposals, a number of electronic document delivery systems are in place, each committed to interoperability facilitated through the application of GEDI defined protocols. GEDI is a practical demonstration of a pragmatic idea.

(Reprinted with permission from the International Publishers Bulletin, No.3, 1992, Vol. VIII (ISSN 0256-6613), Geneva, Switzerland)

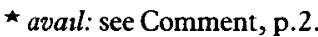

\section{NCTE Resolutions in 1992}

At the annual business meeting of their convention in Louisville, November 1992, directors and members of the National Council of Teachers of English passed resolutions on education issues, among them the need to inform the public and teach students about advertisers' sophisticated techniques of persuasion. They committed NCTE to increased 